\title{
Precise tuning of bacterial translation initiation by non-equilibrium 5'-UTR unfolding observed in single mRNAs
}

\section{Sujay Ray}

University of Michigan-Ann Arbor

\section{Shiba Dandpat}

University of Michigan-Ann Arbor

\section{Surajit Chatterjee}

University of Michigan-Ann Arbor

Nils Walter ( $\nabla$ nwalter@umich.edu )

University of Michigan-Ann Arbor https://orcid.org/0000-0002-7301-1275

\section{Article}

Keywords: 5'-UTR structures, preQ1 riboswitch, Shine-Dalgarno sequence, ribosome binding, singlemolecule kinetics.

Posted Date: June 1st, 2021

DOI: https://doi.org/10.21203/rs.3.rs-551111/v1

License: (9) This work is licensed under a Creative Commons Attribution 4.0 International License. Read Full License 


\section{Precise tuning of bacterial translation initiation by non-}

\section{equilibrium 5'-UTR unfolding observed in single mRNAs}

Sujay Ray†, Shiba S. Dandpat, Surajit Chatterjee and Nils G. Walter*

Single-Molecule Analysis Group, Department of Chemistry and Center for RNA Biomedicine,

University of Michigan, Ann Arbor, MI 48109, USA. *e-mail: nwalter@umich.edu, †Current address:

Wyss Institute for Biologically Inspired Engineering at Harvard University.

KEYWORDS: 5'-UTR structures, preQ $_{1}$ riboswitch, Shine-Dalgarno sequence, ribosome binding, singlemolecule kinetics. 


\section{ABSTRACT}

Noncoding, structured 5' - untranslated regions (5'-UTRs) of messenger RNAs (mRNAs) control translation efficiency by forming structures that can either recruit or repel the ribosome. Here we exploit a bacterial, preQ ${ }_{1}$-sensing translational riboswitch to probe how binding of a small ligand controls binding of the bacterial ribosome to the Shine-Dalgarno (SD) sequence. Combining singlemolecule fluorescence microscopy with mutational analyses, we find that the stability of $30 \mathrm{~S}$ ribosomal subunit binding is inversely correlated with the free energy needed to unfold the $5^{\prime}$-UTR during mRNA accommodation from the standby site to the binding cleft. Ligand binding stabilizes 5'-UTR structure to both antagonize 30 S recruitment and accelerate 30 S dissociation. Depletion of small ribosomal subunit protein S1, known to resolve structured 5'-UTRs, further increases the energetic penalty for mRNA accommodation. The resulting model of rapid standby site exploration followed by gated non-equilibrium unfolding of the 5'-UTR during accommodation provides a mechanistic understanding of translation efficiency. 


\section{Introduction}

5'-Untranslated regions (5'-UTRs) of messenger RNAs (mRNA) are essential for regulating protein expression in all cells ${ }^{1-5}$. Direct interactions between the small, or 30S, ribosomal subunit, and the $5^{\prime}-$ UTR allow modulation of initiation as the rate-limiting step of bacterial mRNA translation ${ }^{5-8}$. The earliest stage of initiation involves two steps as major contributors to overall translation efficiency: reversible binding of the mRNA to a loosely defined "standby site", followed by accommodation into the mRNA binding cleft of the 30 S subunit ${ }^{9-11}$. The cleft-accommodated 30 S ribosomal binding site (RBS) of the mRNA stretches from nucleotides (nt) -18 to +10 relative to the start site (position +1$)^{12}$ and often encompasses a purine-rich region around positions -7 to -4 known as the Shine Dalgarno (SD) sequence ${ }^{13}$. This sequence directly engages with the $3^{\prime}$-terminus of the $16 \mathrm{~S}$ ribosomal RNA (rRNA) of the 30 S subunit - the so-called anti-SD sequence - through base pairing. Translation initiation thus requires unfolding of intrinsic mRNA structure near the start codon, imposing a structure-dependent energetic penalty on translation efficiency ${ }^{13-17}$. Consequently, transcriptomewide studies have shown that an increased translation efficiency of a given mRNA generally correlates with its reduced propensity to form secondary structures near the RBS $15,16,18$.

While the absence of local secondary structure permits efficient translation initiation also on mRNAs that lack an SD sequence ${ }^{19}$, structured RNA motifs such as riboswitches embedded in the $5^{\prime}$ UTR require an SD sequence for efficient gene expression, enabling dynamic regulation ${ }^{14-17,20-22}$. Primarily found in bacteria, riboswitches are cis-regulatory elements typically controlling either transcription or translation ${ }^{15-17}$. Following ligand binding to its aptamer domain, a typical translational riboswitch changes the secondary structure of the downstream expression platform to sequester (part of) the SD sequence, thus repressing translation initiation ${ }^{18}$. 
The extent of riboswitch-mediated translational control varies across the many riboswitch classes discovered to date. In general, larger and more complex riboswitches may entirely abrogate SD and/or start codon access, leading to switch-like ON/OFF behavior ${ }^{23,24}$. In contrast, smaller

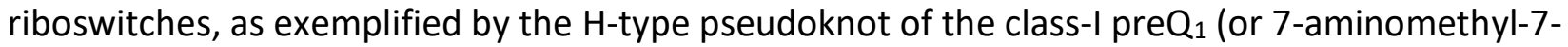
deazaguanine) riboswitch from Thermoanaerobacter tengcongensis (Tte), affect gene expression through only partial SD sequestration (Fig. 1) ${ }^{25-29}$. How binding of a small ligand, which yields only little thermodynamic free energy $(\sim 10 \mathrm{kcal} / \mathrm{mol})^{29,30}$, regulates mRNA accessibility by the much larger bacterial 30S subunit has remained a conundrum. Transcriptome-wide in vivo SHAPE-MaP analysis of general translation initiation supports a model wherein an mRNA first binds to a standby site on the $30 S$ subunit ${ }^{7}$, followed by transient unfolding of $5^{\prime}$-UTR structure during accommodation into the mRNA cleft, imposing a non-equilibrium energetic penalty on translation initiation ${ }^{10,11}$. In the context of riboswitches, these findings suggest that the relative non-equilibrium aptamer unfolding versus $30 \mathrm{~S}$ binding kinetics may play a gateway role in regulation ${ }^{31,32}$, however, the mechanism of this ligand-controlled process has not been observed directly.

In this study, we implement a Single-Molecule Kinetic Analysis of Ribosome Binding (SiM-KARB) assay based on a labeled bacterial 30S subunit to probe 5'-UTR accessibility directly as an important control mechanism of translation initiation by the Tte preQ ${ }_{1}$ riboswitch. Repetitive binding and dissociation of the $30 \mathrm{~S}$ subunit to the riboswitch-hosting mRNA (henceforth referred to as "R-mRNA") reveals short binding events that mutational analyses enable us to assign as non-specific "standby site" interactions, whereas significantly longer binding events represent "cleft-accommodated" interactions where R-mRNA becomes partially unfolded and accommodated into the mRNA binding cleft of the 30 S subunit. We find that both standby and cleft-accommodated binding are similarly 
affected by preQ $_{1}$. Strategic mutations in or near the RBS unveil that increasing aptamer-SD distance and SD:anti-SD base pairing strength both favor initial recognition as well as stable cleftaccommodated binding by the $30 \mathrm{~S}$ subunit. Finally, the effect of RNA secondary structure near the RBS is further verified by depleting ribosomal protein S1, upon which the 30 S subunit shows impaired unfolding of the riboswitch as evidenced by an increased energetic penalty of mRNA accommodation. Taken together, our data support a model wherein the stability of $30 \mathrm{~S}$ binding is inversely related to the non-equilibrium free energy of aptamer unfolding during mRNA accommodation, enabling the ligand to reduce both 305 binding speed and kinetic stability as two distinguishable mechanisms for modulating translation efficiency.

\section{Results}

\section{Riboswitch ligand modulates 30 S binding to the mRNA 5'-UTR}

Prior crystallographic and single-molecule FRET studies of its isolated aptamer domain have suggested that the Tte preQ $_{1}$ riboswitch controls translation initiation by the ligand-induced sequestration of two nucleotides shared between the P2 helix of the pseudoknot and the SD sequence $28,29,33,34$ (Fig. 1a, 1b). The 30 S subunit (Fig. 1c), however, occupies a total of 30 nt in the $5^{\prime}-$ UTR that unfold when stably accommodated into the mRNA binding cleft and are thus considered part of the expression platform (Fig. 1d). We in vitro transcribed a Tte gene-1564 sequence that includes this entire expression platform under the influence of the $\mathrm{preQ}_{1}$ riboswitch, either as a fulllength mRNA (R-mRNA ${ }^{F L}$ ) or as shorter nascent RNA sequences as would emerge during transcription as a platform for binding the first $30 \mathrm{~S}$ subunit that initiates the pioneering round of translation (Fig. 1d) ${ }^{27}$. Exploiting the high sequence conservation and comparable thermodynamics of the SD:anti-SD interactions between Tte and Escherichia coli (Eco; Fig. 1e), we used purified Eco 30S subunits for 
comparing full initiation complex (IC) formation efficiencies on R-mRNAs of different lengths. As expected, we found similar efficiencies for R-mRNA ${ }^{F L}$ and the RNA truncated 30 nt downstream of the start codon (R-mRNA ${ }^{+30}$ ), but observed no IC formation on the RNA lacking both SD sequence and start codon (R-mRNA ${ }^{-11}$; Supplementary Fig. 1). To focus on the role of the 5'-UTR in controlling access of the 30 S subunit without the undesired potential for binding further downstream, we therefore chose the short, initiation-competent R-mRNA ${ }^{+30}$ for our studies.

To characterize the $30 \mathrm{~S}$ binding efficiency of $\mathrm{R}-\mathrm{mRNA}^{+30}$ in response to the addition of preQ ${ }_{1}$ ligand, we performed a radioactive filter-binding assay ${ }^{35}$, wherein the fraction of 30S-bound over total ${ }^{32}$ P-radiolabeled R-mRNA ${ }^{+30}$ is calculated from the blotted, double-filtered dots (Fig. 1f). A time course of the normalized 30S-mRNA complex formation in the absence and presence of a high concentration $(10 \mu \mathrm{M})^{27}$ of $\mathrm{preQ}_{1}$ revealed an $\sim 2$-fold adverse effect of $\mathrm{preQ}_{1}$ on both pre-steady state kinetics and endpoint (Fig. 1f). This level of modulation is very similar to earlier findings that the translation of R-mRNA ${ }^{F L}$ is decreased in vitro by $\sim 40 \%$ at saturating $\operatorname{preQ}_{1}$ concentration ${ }^{27}$, supporting the notion that the ligand significantly affects translation at the initial 30 S binding step. The molecular mechanism of this effect, however, is not revealed by such bulk binding assays.

\section{Riboswitch ligand both antagonizes 30S recruitment and accelerates 30 S dissociation}

To directly evaluate $30 \mathrm{~S}$ binding to $\mathrm{R}-\mathrm{mRNA}^{+30}$ as an important gateway to initiating translation, we developed SiM-KARB, wherein a purified Eco-30S subunit with an extended $16 \mathrm{~S}$ rRNA helix-44 is fluorescently tagged ${ }^{36}$ by hybridization with a doubly (to mitigate photobleaching) Cy5labeled DNA oligonucleotide (Fig. 1c). Repeated binding and dissociation of an excess of these $30 \mathrm{~S}$ subunits in solution (red) to surface-immobilized 3'-Cy3 labeled R-mRNA ${ }^{+30}$ (green) was monitored by 
total internal reflection fluorescence (TIRF) microscopy as transient diffraction-limited co-localization events, performed in the 50 mM Tris-polymix buffer routinely used in translation assays ${ }^{37}$ (Fig. 2a,b; see Methods for details). A mixture of short and long binding events was observed with the presence of preQ $_{1}$ modulating the events (Fig. 2b), supporting two main distinguishable classes of 30S-mRNA interactions, consistent with the known standby and cleft-accommodated binding modes, respectively.

To obtain more quantitative, comparative insights, we pursued several layers of further analysis. First, individual time traces obtained by folding and equilibrating $\mathrm{R}-\mathrm{mRNA} \mathrm{A}^{+30}$ in the presence of varying concentrations of $\mathrm{preQ}_{1}$ were idealized into two-state Hidden Markov Models (HMM, Fig. 2b). The HMMs were then used to extract the dwell times during which 305 was either unbound ( $\left.\tau_{\text {unbound }}\right)$ or bound $\left(\tau_{\text {bound }}\right)$, which both were best fit globally with double-exponential functions ${ }^{38,39}$ revealing each two rate constants: 305 binding rate constants $k_{o n, f a s t}$ and $k_{o n, s l o w}$, and 30 dissociation rate constants $k_{\text {off,fast }}$ and $k_{\text {off,slow, }}$ respectively. Of these, the slow $k_{\text {on, slow }}$ and $k_{\text {off,slow }}$ represented $\sim 30$ $60 \%$ and $\sim 70-90 \%$, respectively, of all binding events and were found to be most significantly affected by the addition of preQ $_{1}$, with $k_{\text {on,slow }}$ decreasing and $k_{\text {off,slow }}$ increasing (Fig. $2 \mathrm{c}$ and Supplementary Table S2 A,B). Of note, while dual Cy5-labeling prolonged the observation of bound 30S subunits, we corrected all rate constants for photobleaching of both fluorophores (Supplementary Fig. 2). The observed $\sim 27 \%$ deceleration of $30 \mathrm{~S}$ binding with increasing preQ $_{1}$ (from $k_{\text {on, slow }}=0.33 \pm 0.04 \times 10^{6} \mathrm{M}^{-}$ ${ }^{1} \mathrm{~s}^{-1}$ of relative amplitude $=89 \pm 4 \%$; to $0.24 \pm 0.03 \times 10^{6} \mathrm{M}^{-1} \mathrm{~s}^{-1}$ of $96 \pm 8 \%$; Fig. $2 \mathrm{c}$, Supplementary Fig. S3 and Supplementary Table S2A) is consistent with a model of partial SD sequestration upon preQ ${ }_{1}$ induced folding of the riboswitch. By contrast, the 3-fold acceleration of 30 S dissociation (from $k_{\text {off,slow }}=0.002 \pm 0.001 \mathrm{~s}^{-1}$ of relative amplitude $58 \pm 8 \%$; to $0.006 \pm 0.002 \mathrm{~s}^{-1}$ of $41 \pm 8 \%$; Fig. $2 \mathrm{c}$, 
Supplementary Fig. S3 and Supplementary Table S2A) suggests an additional effect of preQ ${ }_{1}$ on dissociation of the assembled 30S-R-mRNA ${ }^{+30}$ complex. Global fitting of the two ligand dependencies with non-cooperative Hill equations yielded a half-saturation point of $\sim 48 \mathrm{nM}$ preQ ${ }_{1}$ (Fig. $2 \mathrm{C}$ ), in reasonable agreement with measurements of ligand binding to the isolated riboswitch ${ }^{28,29}$ and demonstrating that $\mathrm{R}-\mathrm{mRNA}^{+30}$ is saturated at $1 \mu \mathrm{M}$ preQ ${ }_{1}$.

Second, we asked how individual 30 S binding kinetics change for individual R-mRNA ${ }^{+30}$ molecules when folded with $1 \mu \mathrm{M}_{\mathrm{preQ}}$ present. To this end, individual trace HMMs were arranged into rastergrams sorted by their relative accessibility to the $30 \mathrm{~S}$ subunit over the entire trace (Fig. $2 \mathrm{~d}$ ). Based on a threshold bound time derived from the $k_{\text {off,fast }}$ and $k_{\text {off,slow }}$ dissociation rate constants (Materials and Methods), we categorized individual binding events in the rastergram as either short (red) or long (blue) (Fig. 2d). These rastergrams indicate that each R-mRNA ${ }^{+30}$ molecule interacts quite distinctly with 30 s subunits and shows long-term retention of its 30 s occupancy behavior. As expected from an SD sequestration model, the number of total $30 \mathrm{~S}$ binding events decreases upon addition of saturating $\mathrm{preQ}_{1}$ ligand, by $\sim 17 \%$ (Fig. $2 \mathrm{~d}$ ). Moreover, while the shorter binding events were less affected $(\sim 6 \%)$, the long binding events reduced by $\sim 33 \%$ with the addition of preQ1 (supporting table S3), suggesting that the longer, cleft accommodated binding of the $30 \mathrm{~S}$ are primarily disfavored by preQ1 induced riboswitch folding.

Lastly, inspired by previous studies equally suggesting that 305 occupancy behaviors of individual mRNA molecules may be retained long-term, in aggregate leading to a significant downregulation of protein expression ${ }^{27}$, we plotted probability histograms that bin the total fraction of time single R-mRNA ${ }^{+30}$ molecules remain 30 S associated within our experimental time window of 1,000 s (Supplementary Fig. 4B). From the combined histogram of all datasets (Supplementary Fig. 
4A), a 30 S occupancy ranking of individual molecules as Low (L, fractional 30 S bound time $<0.10)$, Medium (M, fractional 30S bound time between 0.10 and 0.20$)$ or High $(\mathrm{H}$, fractional $30 \mathrm{~S}$ bound time $>0.20$ ) was derived. In the absence of $\operatorname{preQ}_{1}, 30 \pm 4 \%, 44 \pm 3 \%$ and $26 \pm 5 \%$ of molecules show $L, M$, and $\mathrm{H}$ occupancy, respectively; whereas upon addition of saturating $1 \mu \mathrm{M}$ preQ ${ }_{1}$, a large majority of the molecular population ( $81 \pm 2 \%$ ) exhibits L occupancy, at the expense of both the $M(13 \pm 2 \%$ ) and $\mathrm{H}(6 \pm 2 \%)$ occupancies (Fig. $2 \mathrm{e})$.

These findings suggest that individual molecules belong to subpopulations that respond individually to $\mathrm{preQ}_{1}$ addition with a common trend in which 305 binding becomes suppressed, thereby lowering the probability of translation initiation. Our data also support the model of two binding interactions between R-mRNA ${ }^{+30}$ and 30 S subunits, with weak standby site binding and more stable (longer-lived) binding events upon cleft accommodation, both of which are similarly impacted by the presence of preQ ${ }_{1}$. Finally, the ligand not only competes with $30 \mathrm{~S}$ for binding to the RNA pseudoknot, but also actively accelerates dissociation of 30 S subunits already bound to R-mRNA ${ }^{+30}$.

\section{The riboswitch-hosting mRNA dynamically adapts to changes in preQ ${ }_{1}$ concentration}

The persistence of individual R-mRNA +30 in their equilibrium interaction with 30 S subunits raises the question to what extent $30 \mathrm{~S}$ binding to $\mathrm{R}-\mathrm{mRNA} \mathrm{A}^{+30}$ changes in response to sudden, nonequilibrium variations in its environment, as may occur in the bacterial cells due to, e.g., environmental stresses ${ }^{40}$. To address this question, we used a non-equilibrium preQ ${ }_{1}$ "ligand-jump" experiment from zero to saturating ligand while tracking $30 \mathrm{~S}$ binding (Fig. 3a, Materials and methods $)^{27,41}$. Consistent with our equilibrium experiments, upon preQ ${ }_{1}$ addition we observed a general reduction in $30 \mathrm{~S}$ binding frequency (Fig. 3a top and middle) and a decrease in the $30 \mathrm{~S}$ bound 
time (Fig. 3a, bottom). Overall, the resulting 30\% decrease in $k_{\text {on, slow }}$ and $\sim 3$ fold increase in $k_{\text {off,slow }}$ (Supplementary Fig. 6) suggest that $\mathrm{preQ}_{1}$ binding in situ has similar effects on $30 \mathrm{~S}$ association of R$\mathrm{mRNA}^{+30}$ as does its equilibrium binding, both antagonizing $30 \mathrm{~S}$ recruitment and accelerating dissociation.

Next, we analyzed the probability distribution of 305 fractional bound times. In the absence of preQ $_{1}, 46 \pm 3 \%$ of R-mRNA ${ }^{+30}$ molecules showed L-type 30 S occupancy, while $30 \pm 3 \%$ and $24 \pm 3 \%$ of molecules showed $\mathrm{M}$ and $\mathrm{H}$ accessibility, respectively (Fig. 3b). By contrast, after addition of saturating preQ $_{1} \mathrm{mRNA}$ molecules predominantly exhibited reduced accessibility $(82 \pm 2 \% \mathrm{~L}, 12 \pm 2 \%$ $\mathrm{M}$ and $6 \pm 1 \% \mathrm{~L}$, Fig. 3c). This shift from high to low 30 S occupancy of the same molecules in response to $\mathrm{preQ}_{1}$ further supports that dynamic ligand binding induces the same repulsion and destabilization of 305 subunit binding observed under equilibrium conditions.

Finally, a rastergram allowed us to directly compare the mRNA accessibility in the absence and presence of preQ $_{1}$ (Fig. 3d). 48\% of molecules responded to the addition of preQ ${ }_{1}$ with a significant reduction in 30S occupancy (Supplementary Table S4A). We also found that the short standby site interactions were reduced by $\sim 30 \%$, whereas the longer cleft-accommodated interactions were reduced even more profoundly, by $\sim 60 \%$, underscoring the large effect of $\mathrm{preQ}_{1}$ on particularly cleftaccommodated interactions (Supplementary Table S4B). Still, 46\% of molecules remained in the similar 30S occupancy and did not significantly respond to the addition of preQ ${ }_{1}$. Only a small ( $\left.\sim \%\right)$ population transitioned from a less to a higher $30 \mathrm{~S}$ occupancy. The latter observation suggests that, under non-equilibrium conditions, $\mathrm{preQ}_{1}$ in some cases may promote the refolding of an $\mathrm{mRNA}$, as 
was observed previously ${ }^{27}$. On the other end of the behavioral spectrum, some $15 \%$ of R-mRNA ${ }^{+30}$ molecules became completely inaccessible to $30 \mathrm{~S}$ binding.

Overall, these observations underscore the dynamic nature of the significant response of the riboswitch to ligand binding, rending $30 \mathrm{~S}$ binding and thus translation efficiency highly adaptive to environmental cues.

\section{Riboswitch pseudoknot proximity and SD sequence strength independently control 30 S binding}

In bacteria, the SD sequence is not obligatory for translation initiation ${ }^{42,43}$. While only $0.7 \%$ of mRNAs are thought to be leaderless in $E$. coli ${ }^{44}$, other bacteria harbor up to $72 \%$ leaderless mRNAs ${ }^{45}$. The $\mathrm{preQ}_{1}$ riboswitch and the SD sequence overlap such that $\mathrm{preQ}_{1}$ binding promotes $\mathrm{P} 2$ helix formation of the aptamer pseudoknot, sequestering two nucleotides of the SD sequence ${ }^{29}$ (Fig. 1a,b). Taken together, these observations raise the question of how sequestering only $2 \mathrm{nt}$ can have the profound effect on $30 \mathrm{~S}$ binding we observe ${ }^{29}$. To address this question, we next created a systematic set of R-mRNA ${ }^{+30}$ mutants that systematically decouple the aptamer and SD sequence.

In the first set, the aptamer and SD sequence were increasingly separated by inserting 1-6 nt between them while maintaining the 8-nt long wild-type (WT) SD sequence (Fig. 4a, constructs I1S816S8; Supplementary Table S1 for the list of sequences). A second set of mutations aimed to increasingly weaken the SD:anti-SD interaction by shortening the SD length from 8 to zero nt while maintaining an unstructured 4-nt UAUA insertion between the aptamer and the remaining SD (Fig. 4e,constructs 14S8 to 14S0; Supplementary Table S1 for the list of sequences). All mutants retained all 
nucleotides participating in the pseudoknotted aptamer structure, and the WT would be annotated $12 \mathrm{~S} 8$ in this nomenclature.

Strikingly, using our equilibrium SiM-KARB assay, we found a $k_{\text {on, slow }}$ of $0.25 \pm 0.009 \times 10^{6} \mathrm{M}^{-1} \mathrm{~S}^{-}$

${ }^{1}$ for IIS8 in the absence of $\mathrm{preQ}_{1}$, within error the same value as the WT displays at saturating $\mathrm{preQ}_{1}$ (0.24 $\pm 0.01 \times 10^{6} \mathrm{M}^{-1} \mathrm{~s}^{-1} ;$ Fig. 4b, Supplementary Table S6). This mutant I1S8, as well as I4S8, showed only a minimal effect of preQ $_{1}$ on the 30 S binding kinetics (Supplementary Fig. 7). That is, when the aptamer is entirely decoupled (removing any overlap) and separated by $1 \mathrm{nt}$ from the canonical 8-nt SD sequence, it represses 30S binding independently of preQ $_{1}$ as efficiently as the WT does only when fully folded with preQ ${ }_{1}$. Since the aptamer's P2 helix forms only partially in the absence of $\mathrm{preQ}_{1}{ }^{29}$, this repression does not depend on occlusion of part of the SD sequence, but instead must arise from the steric hindrance the pre-folded, preQ $_{1}$-free (or apo) aptamer structure exerts on the $30 \mathrm{~S}$ subunit. In support of this hypothesis, $k_{\text {on, slow }}$ values of $0.34 \pm 0.007 \times 10^{6} \mathrm{M}^{-1} \mathrm{~s}^{-1}$ and $0.36 \pm 0.006 \times 10^{6} \mathrm{M}^{-1} \mathrm{~s}^{-1}$ were recovered in the absence of $\mathrm{preQ}_{1}$ once the SD was moved 6 and $4 \mathrm{nt}$ apart from the SD in mutants 1658 and 14S8, respectively (Fig. 4b, Supplementary Fig. 8 and Table S6), equivalent to the value of $0.33 \pm 0.02 \times 10^{6} \mathrm{M}^{-1} \mathrm{~s}^{-1}$ for the WT without preQ $_{1}$. A mutant of intermittent 2-nt distance (I2S8) showed an intermediate increase of $k_{\text {on, slow, also in the absence of preQ }}$ (Fig. $4 \mathrm{~b}$, Supplementary Fig. 8 and Table S6), suggesting that the 30S subunit is highly sensitive to the precise distance of the partially formed P2 helix from the SD sequence. A rastergram plot confirmed this trend with an $\sim 240 \%$ increase in short standby binding events and an $\sim 7 \%$ increase in long binding events of I6S8 relative to I1S8, showing that as the SD sequence moved away from the steric hindrance of aptamer, it became more accessible for 30S to bind (Fig. 4c, Supplementary Table S7). Similarly, the $30 \mathrm{~S}$ occupancy of single mRNA molecules was low with the aptamer placed proximal to 
the SD sequence in IIS8 (11 $\pm 1 \% \mathrm{H}, 15 \pm 1 \% \mathrm{M}$, and $74 \pm 1 \%$ L occupancy; Fig. $4 \mathrm{~d})$ and increased when the aptamer was more spaced out, as in I6S8 $(24 \pm 2 \% \mathrm{H}, 33 \pm 2 \% \mathrm{M}$ and $43 \pm 2 \% \mathrm{~L}$; Fig. $4 \mathrm{H})$.

Taken together, these observations reveal that the pre-folded apo-aptamer represses $30 \mathrm{~S}$ association sterically during the earliest stage of translation initiation, whereas spacing the aptamer's P2 helix as little as 4 nt from the SD sequence alleviates this steric hindrance of 30S binding entirely. This finding reveals a surprisingly limited role of the SD sequence itself in effecting riboswitchmediated repression of translation initiation. Notably, the dissociation rate constants $k_{\text {off,slow }}$ of this entire set of mutants from I1S8 to I6S8 in the absence of preQ ${ }_{1}$ remains elevated, closer to that of the WT in the presence of preQ $_{1}$ than its absence (Fig. 4b), suggesting that the mRNA-30S complex, once formed, may be sensitive to secondary structure of the mRNA even further upstream.

To understand the role of the SD sequence, if any, we investigated our second set of mutants, I4S8 to I4S0 (Fig. 4e, Supplementary Fig. 8 and Table S6). Using our SiM-KARB assay under equilibrium conditions in the absence of preQ $_{1}$, we found $14 S^{\prime}$ 's $k_{\text {on, slow }}$ value of $0.36 \pm 0.006 \times 10^{6} \mathrm{M}^{-1} \mathrm{~s}^{-1}$ to decrease to $0.26 \pm 0.008 \times 10^{6} \mathrm{M}^{-1} \mathrm{~s}^{-1}$ for $14 \mathrm{SO}$ as the number of complementary SD:anti-SD base pairs decreased from eight to zero (Fig. 4f, Supplementary Fig. 8 and Table S8). This $~ 35 \%$ decrease in binding rate constant, and gradual decrease at intermediate SD sequence lengths (Fig. 4f), underscores that the mRNA's SD sequence is an important 30 S recruiting factor. Notably, the $30 \mathrm{~S}$ subunit still binds to I4SO even in the entire absence of the SD sequence, which corroborates the notion that leaderless mRNAs can function in translation ${ }^{42,43}$. We also observed that the $k_{\text {on, slow }}$ for I4SO in the absence of preQ $_{1}$ comes close to that of the WT in its presence (Fig. 4f), providing evidence that $\mathrm{preQ}_{1}$ binding to the WT aptamer suppresses access to the SD sequence completely, even though the $30 \mathrm{~S}$ subunit can still bind elsewhere on our short mRNA. Of note, the dissociation 
rate constants $k_{\text {off, slow }}$ of this set of mutants from $14 \mathrm{~S} 8$ to $14 \mathrm{SO}$ in the absence of preQ ${ }_{1}$ again remains closer to the elevated value of the WT in the presence of preQ ${ }_{1}$ (Fig. 4f), consistent with the notion of mRNA-30S complex sensitivity to proximal mRNA secondary structure. A comparison of the rastergrams for 14S8 and I4S0 showed that, while the cleft-accommodated (long) binding events were reduced by $\sim 52 \%$, the short standby bindings were reduced even more, by $\sim 45 \%$ (Fig. $4 \mathrm{~g}$, Supplementary Table S7). Finally, the fractional 30S occupancy histograms showed that the mRNA accessibility progressively decreases from $17 \pm 2 \% \mathrm{H}, 23 \pm 2 \% \mathrm{M}$, and $60 \pm 2 \% \mathrm{~L}$ population for $14 \mathrm{~S} 8$ to $10 \pm 2 \% \mathrm{H}, 8 \pm 2 \% \mathrm{M}$, and $82 \pm 2 \% \mathrm{~L}$ for $14 \mathrm{SO}$ (Fig. $4 \mathrm{~h}$ ).

Taken together, our observations from mutations designed to systematically uncouple aptamer and SD sequence effects indicate that ${ }^{43}$ the absence of steric hindrance from proximal secondary structure and a strong SD:anti-SD interaction are two main, independent 30S recruitment factors. That is, while $\mathrm{preQ}_{1}$ binding to the aptamer only sequesters two nucleotides of the SD sequence, the riboswitch affects $30 \mathrm{~S}$ recruitment through both partial occlusion of the SD sequence upon ligand binding and, unexpectedly, its inherent secondary structure independent of ligand.

\section{Ribosomal protein S1 unfolds the riboswitch to both facilitate and stabilize 30 S binding}

Our data so far have shown that preQ $_{1}$ binding to WT R-mRNA ${ }^{+30}$ reduces SD sequence accessibility and thereby both slows and destabilizes $30 \mathrm{~S}$ binding. Ribosomal protein S1 is known to enhance $30 \mathrm{~S}$ binding by many mRNAs with structured 5'-UTRs ${ }^{46,47}$. S1's three central domains bind and resolve RNA structures, including that of the Tte preQ1 riboswitch ${ }^{33}$, to help accommodate them into the mRNA binding cleft (Fig. 5a) ${ }^{48}$. S1 loosely binds to the 30 S ribosomal subunit and can be depleted 
during purification ( $\triangle \mathrm{S} 1-30 \mathrm{~S})$ and subsequently be reconstituted by adding recombinant S1 (Methods, Supplementary Fig. 9). This approach gives us as tool to test our SD accessibility model further.

Our SiM-KARB assay demonstrated that the $\triangle \mathrm{S} 1-30 \mathrm{~S}$ subunit binds to R-mRNA ${ }^{+30}$ with diminished frequency and stability (Fig. 5b); stable binding was recovered only by reconstituting $\Delta$ S130S with purified S1 (Supplementary Fig. 10). Accordingly, the mRNA occupancy histograms showed very low accessibility of individual R-mRNA ${ }^{+30}$ molecules to $\Delta$ S1-30S, counteracted by S1 reconstitution (Fig. 5c). Plotting the $H, M$ and $L$ population fractions we found that the L population decreased from $70 \pm 8 \%$ for $\Delta$ S1-30S to $29 \pm 4 \%$ for WT-30S, while the M and L population increased from $9 \pm 2 \%$ and $21 \pm 7 \%$ for $\Delta$ S1-30S to $44 \pm 3 \%$ and $26 \pm 5 \%$, respectively, for WT-30S (Fig. $5 d$ ). These findings suggest that 5'-UTR access is strongly facilitated by S1-mediated RNA unfolding.

Accordingly, we observed a $k_{\text {on, slow }}$ of $0.24 \pm 0.02 \times 10^{6} \mathrm{M}^{-1} \mathrm{~s}^{-1}$ for $\Delta \mathrm{S} 1-30 \mathrm{~S}, \sim 1.4$-fold lower than that for WT-30S. In contrast, the $k_{\text {off,slow }}$ of $0.01 \pm 0.02 \mathrm{~s}^{-1}$ for $\Delta$ S1-30S was elevated $\sim 2$-fold over WT30S (Fig. 5d). That is, not only does S1 unfold the 5'-UTR to facilitate initial 30 S binding (i.e., increases

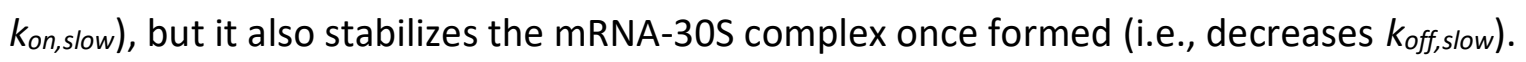

Stoichiometric (1:1) reconstitution of $\Delta \mathrm{S} 1-30 \mathrm{~S}$ with recombinant S1 recovered particularly the $k_{\text {off, slow }}$ of WT-30S, whereas $k_{\text {on,slow }}$ is restored to a lesser degree (Fig. $5 \mathrm{~d}$ ), possibly because free S1 protein in solution coats the pseudoknot, thereby partially blocking 305 recruitment.

These results underscore, first, the important role of S1 protein in accommodating structured RNAs into the 30 S subunit's mRNA binding cleft to initiate translation and, second, further support our model that proximal mRNA structure both antagonizes 305 recruitment and accelerates its dissociation. 
Initiation factors only slightly facilitate 305 binding, independent of the 5'-UTR structure

Lastly, we explored how initiation factors (IFs) and initiator fMet-tRNA ${ }^{f M e t}$, known to be essential for translation initiation in vivo, impact 30 S binding in the context of $5^{\prime}$-UTR structure ${ }^{49,50}$. While each IF governs a specific function in the initiation process 51,52 , we asked to what extent they together influence 30 S binding by adding recombinant IF1, IF2, and IF3, and fMet-tRNA ${ }^{\text {fMet }}$ with the 30 S into our SiM-KARB assay (Fig. 5e) and examining the kinetics of $30 \mathrm{~S}$ binding to $R-m R N A^{+30}$. We found that the fractional 30S occupancy in the presence of IFs and tRNA rose from $11 \pm 3 \% \mathrm{H}, 26 \pm 2 \% \mathrm{M}$, and $63 \pm 4 \% \mathrm{~L}$ in the absence of $\mathrm{preQ}_{1}$ to $1 \pm 1 \% \mathrm{H}, 4 \pm 2 \% \mathrm{M}$, and $95 \pm 2 \% \mathrm{~L}$ at saturating $\mathrm{preQ}_{1}$, not drastically different from that in the absence of IFs and tRNA (compare Figs. 2e and 5f). In the presence of IFs and tRNA $\mathrm{fMet}^{\text {, }}, k_{\text {on, slow }}$ increased to $0.39 \pm 0.01 \times 10^{6} \mathrm{M}^{-1} \mathrm{~s}^{-1}$ from $0.33 \pm 0.04 \times 10^{6} \mathrm{M}^{-1} \mathrm{~s}^{-1}$ in their absence; whereas upon addition of $\operatorname{preQ}_{1}, k_{\text {on, slow }}$ only slightly increased in the presence of IFs and tRNA ${ }^{\text {fMet }}$ to $0.27 \pm 0.04 \times 10^{6} \mathrm{M}^{-1} \mathrm{~s}^{-1}$ from $0.24 \pm 0.03 \times 10^{6} \mathrm{M}^{-1} \mathrm{~s}^{-1}$ in their absence; finally, $k_{\text {off, slow }}$ remained within error unchanged (Fig. 5g and Supplementary Table S13B).

In summary, we thus find that IFs and tRNA ${ }^{\text {fMet }}$ slightly increase $k_{\text {on, slow }}$ over the values in their absence, equally in the presence and absence of $\mathrm{preQ}_{1}$, while not significantly affecting $k_{\text {off,slow. }}$ These observations indicate that the cofactors play a small, supportive role in the earliest stage of translation initiation as probed by $30 \mathrm{~S}$ binding and exert it equally on the pre-folded apo-riboswitch and the fully folded preQ 1 -bound riboswitch.

\section{Discussion}

By examining 30S-mRNA interactions at the earliest stage of translation initiation, we reveal here how a small, but strategically placed 5'-UTR structure can influence gene expression. We find that $30 \mathrm{~S}$ 
ribosomal subunit binding to the mRNA downstream of an $\mathrm{H}$-type pseudoknotted riboswitch is controlled both by the RNA's secondary structure, independently of ligand binding to the riboswitch aptamer, and by the occlusion of the SD sequence upon ligand binding. Moreover, pre ${ }_{1}$ binding itself exerts two distinct effects; first, antagonizing 30 S recruitment (i.e., slowing down $30 \mathrm{~S}$ binding) and, second, destabilizing the 30S-mRNA complex once formed (i.e., accelerating 30S dissociation), giving the mRNA multiple layers of control over translation. Our mutational studies highlight the importance of the SD:anti-SD base pairing to initiate translation ${ }^{53,54}$, although it turns out to be only one of several mechanisms by which a translational riboswitch governs gene expression of its hosting mRNA. Such a multi-pronged effect leverages binding of a small ligand into efficient regulation of translation initiation by the much larger bacterial 30 s subunit.

Upon decoupling secondary structure and SD sequence, we further find that the precise distance of the aptamer from the SD sequence is a key determinant of initial recruitment of the $30 \mathrm{~S}$ subunit, with a distance of as small as 4 nt eliminating the aptamer's influence on $30 \mathrm{~S}$ binding. While the cleft-accommodated mRNA is footprinted from nucleotides (nt) -18 to +10 on the 30 S subunit, exceeding the SD sequence by 10 nt on the 5 ' side, architecturally the segment of mRNA upstream of the SD sequence is rather solvent exposed ${ }^{12}$, rationalizing how steric clashes rapidly diminish on a length scale shorter than the footprint.

Perhaps most strikingly, we find clear evidence for short-lived standby site exploration, sometimes followed by unfolding of the riboswitch for a longer-lived mRNA cleft accommodation, leading to the model in Fig. 6a. Consequently, the riboswitch ligand and ribosomal protein S1 counteract in that preQ $_{1}$ tightens the pseudoknot fold while S1 unties it to enhance mRNA cleft accommodation (Fig. 6a). Based on our kinetic SiM-KARB measurements, we then can predict a free 
energy model for the non-equilibrium unfolding of the riboswitch (Fig. 6b, Supplementary Fig. 12). The initial binding energy into the standby site is very similar in the absence and presence of preQ ${ }_{1}(-$ $3.6 \mathrm{kcal} / \mathrm{mol}$ ). Successive mRNA accommodation by unfolding the mRNA is a spontaneous process yielding $-0.5 \mathrm{kcal} / \mathrm{mol}$ energy in the absence of $\mathrm{preQ}_{1}$, whereas with $\mathrm{preQ}_{1}$ bound the $5^{\prime}-\mathrm{UTR}$ is more stably folded and requires $+0.9 \mathrm{kcal} / \mathrm{mol}$ to unfold. The net energetic penalty for cleftaccommodation of the mRNA in the presence of $\mathrm{preQ}_{1}$ is thus $1.4 \mathrm{kcal} / \mathrm{mol}$. This small penalty equivalent to a couple of hydrogen bonds - is a reflection of the exchange of two base pairs in helix P2 of the riboswitch for two base pairs of SD:anti-SD interaction (Fig. 1). Due to the multi-pronged effects of the riboswitch on both $30 \mathrm{~S}$ binding and dissociation, this seemingly small effect suffices for the riboswitch ligand to have significant leverage over translation initiation (Fig. 6a).

The leveraging we discovered here of a local, small-scale binding event into the global, largescale functional process of translation initiation appears to be a recurring theme in RNA biology. For example, in a recent study of a $\mathrm{Mn}^{2+}$ sensing riboswitch, a single metal ion binding at the docking core of an RNA four-way junction was shown to alter the global structure to affect transcription ${ }^{55}$. Furthermore, highly structured motifs around the RBS of an mRNA often restrict 30S recruitment to control translation efficiency ${ }^{7,11}$. The classical model of translation initiation on structured mRNAs invoked an equilibrium thermodynamic process, wherein the $5^{\prime}$-UTR is remodeled into low-energy structures by successive unfolding and refolding that then are accommodated into the mRNA binding cleft of the $30 \mathrm{~S}$ subunit ${ }^{56}$. Recent transcriptome-wide structural studies have refined this model and shown that a non-equilibrium kinetic competition between mRNA unfolding and $30 \mathrm{~S}$ dissociation governs translation efficiency ${ }^{7}$. We demonstrate here that this kinetic competition provides leverage to riboswitches to modulate translation efficiency, even in cases where a small ligand (such as preQ ${ }_{1}$ ) 
only provides limited thermodynamic power. Once an mRNA has repelled a 30S subunit, the competition between mRNAs in the bacterial cell dictates that free subunits initiate translation elsewhere, whereas the unoccupied mRNA may fall prey to ribonucleases ${ }^{32}$.

In bacteria, translation efficiency as dictated by secondary structure around the RBS plays a significant role in determining the fate of an $\mathrm{mRNA}^{57-59}$. As initiation complexes form, commitment to translation increases while the vulnerability to mRNA decay decreases, as mediated by the bacterial degradosome, $\mathrm{Hfq}$, and Rho ${ }^{32,60-62}$. Consequently, the evolutionary pressure is high to evolve a finetuned kinetic modulation of initiation complex formation as a key regulator of global translational activity. The insights presented here will therefore provide an opening for the development of novel antibacterial drugs that derail this finely tuned machinery.

\section{Online content}

Any methods, additional references, Nature Research reporting summaries, source data, extended data, supplementary information, acknowledgements, peer review information; details of author contributions and competing interests; and statements of data and code availability are available at...

\section{Acknowledgments}

This work was supported by NIH grants R01 GM062357 and MIRA R35 GM131922 (to N.G.W.). We thank Dr. Joseph (Jody) Puglisi at Stanford University for providing a modified 30S ribosomal subunit suitable for labeling and Ms. Hailey Blinkiewicz for technical assistance. 


\section{Author contributions}

S.R. and N.G.W. designed the study. S.R., S.D. and S.C. performed the experiments and analyzed the data. S.R., S.D., S.C. and N.G.W. wrote the manuscript.

\section{Competing interests}

The authors declare no competing interests.

\section{Additional information}

Supplementary information is available for this paper at ...

Reprints and permissions information is available at www.nature.com/reprints.

Code availability statement: All custom codes used in this study are available from the corresponding author upon reasonable request.

Data availability statement: The data that support the findings of this study are available from the corresponding author upon reasonable request.

Correspondence and requests for materials should be addressed to N.G.W. (nwalter@umich.edu). 


\section{References}

1. Araujo, P.R. et al. Before It Gets Started: Regulating Translation at the 5' UTR. Comp Funct Genomics 2012, 475731 (2012).

2. Dvir, S. et al. Deciphering the rules by which 5'-UTR sequences affect protein expression in yeast. Proc Natl Acad Sci U S A 110, E2792-801 (2013).

3. Leppek, K., Das, R. \& Barna, M. Functional 5' UTR mRNA structures in eukaryotic translation regulation and how to find them. Nat Rev Mol Cell Biol 19, 158-174 (2018).

4. Braun, F., Durand, S. \& Condon, C. Initiating ribosomes and a 5'/3'-UTR interaction control ribonuclease action to tightly couple B. subtilis hbs mRNA stability with translation. Nucleic Acids Res 45, 11386-11400 (2017).

5. Melnikov, S. et al. One core, two shells: bacterial and eukaryotic ribosomes. Nat Struct Mol Biol 19, 560-7 (2012).

6. Na, D., Lee, S. \& Lee, D. Mathematical modeling of translation initiation for the estimation of its efficiency to computationally design mRNA sequences with desired expression levels in prokaryotes. BMC Syst Biol 4, 71 (2010).

7. Mustoe, A.M. et al. Pervasive Regulatory Functions of mRNA Structure Revealed by HighResolution SHAPE Probing. Cell 173, 181-195.e18 (2018).

8. Tuller, T. \& Zur, H. Multiple roles of the coding sequence $5^{\prime}$ end in gene expression regulation. Nucleic Acids Res 43, 13-28 (2015).

9. Andreeva, I., Belardinelli, R. \& Rodnina, M.V. Translation initiation in bacterial polysomes through ribosome loading on a standby site on a highly translated mRNA. Proc Natl Acad Sci U $S$ A 115, 4411-4416 (2018).

10. de Smit, M.H. \& van Duin, J. Translational Standby Sites: How Ribosomes May Deal with the Rapid Folding Kinetics of mRNA. Journal of Molecular Biology 331, 737-743 (2003).

11. Mustoe, A.M., Corley, M., Laederach, A. \& Weeks, K.M. Messenger RNA Structure Regulates Translation Initiation: A Mechanism Exploited from Bacteria to Humans. Biochemistry 57, 3537-3539 (2018).

12. Culver, G.M. Meanderings of the mRNA through the ribosome. Structure 9, 751-8 (2001).

13. Shine, J. \& Dalgarno, L. Terminal-sequence analysis of bacterial ribosomal RNA. Correlation between the 3'-terminal-polypyrimidine sequence of 16-S RNA and translational specificity of the ribosome. Eur J Biochem 57, 221-30 (1975).

14. Nudler, E. \& Mironov, A.S. The riboswitch control of bacterial metabolism. Trends Biochem Sci 29, 11-7 (2004).

15. Breaker, R.R. Prospects for riboswitch discovery and analysis. Mol Cell 43, 867-79 (2011).

16. Winkler, W.C., Nahvi, A., Sudarsan, N., Barrick, J.E. \& Breaker, R.R. An mRNA structure that controls gene expression by binding S-adenosylmethionine. Nat Struct Biol 10, 701-7 (2003).

17. Winkler, W., Nahvi, A. \& Breaker, R.R. Thiamine derivatives bind messenger RNAs directly to regulate bacterial gene expression. Nature 419, 952-6 (2002).

18. Barrick, J.E. \& Breaker, R.R. The distributions, mechanisms, and structures of metabolitebinding riboswitches. Genome Biol 8, R239 (2007).

19. Scharff, L.B., Childs, L., Walther, D. \& Bock, R. Local absence of secondary structure permits translation of mRNAs that lack ribosome-binding sites. PLoS genetics 7, e1002155-e1002155 (2011). 
20. Winkler, W.C., Nahvi, A., Roth, A., Collins, J.A. \& Breaker, R.R. Control of gene expression by a natural metabolite-responsive ribozyme. Nature 428, 281-6 (2004).

21. Winkler, W.C. \& Breaker, R.R. Regulation of bacterial gene expression by riboswitches. Annu Rev Microbiol 59, 487-517 (2005).

22. Mandal, M. \& Breaker, R.R. Gene regulation by riboswitches. Nat Rev Mol Cell Biol 5, 451-63 (2004).

23. Fuchs, R.T., Grundy, F.J. \& Henkin, T.M. S-adenosylmethionine directly inhibits binding of 30S ribosomal subunits to the SMK box translational riboswitch RNA. Proc Natl Acad Sci U SA 104, 4876-80 (2007).

24. Haller, A., Altman, R.B., Souliere, M.F., Blanchard, S.C. \& Micura, R. Folding and ligand recognition of the TPP riboswitch aptamer at single-molecule resolution. Proc Natl Acad Sci U $S$ A 110, 4188-93 (2013).

25. Eichhorn, C.D., Kang, M. \& Feigon, J. Structure and function of preQ(1) riboswitches. Biochim Biophys Acta 1839, 939-950 (2014).

26. Kim, J.N. \& Breaker, R.R. Purine sensing by riboswitches. Biol Cell 100, 1-11 (2008).

27. Rinaldi, A.J., Lund, P.E., Blanco, M.R. \& Walter, N.G. The Shine-Dalgarno sequence of riboswitch-regulated single mRNAs shows ligand-dependent accessibility bursts. Nat Commun 7, 8976 (2016).

28. Jenkins, J.L., Krucinska, J., McCarty, R.M., Bandarian, V. \& Wedekind, J.E. Comparison of a preQ1 riboswitch aptamer in metabolite-bound and free states with implications for gene regulation. J Biol Chem 286, 24626-37 (2011).

29. Suddala, K.C. et al. Single transcriptional and translational preQ $_{1}$ riboswitches adopt similar pre-folded ensembles that follow distinct folding pathways into the same ligand-bound structure. Nucleic Acids Res 41, 10462-75 (2013).

30. Liberman, J.A., Bogue, J.T., Jenkins, J.L., Salim, M. \& Wedekind, J.E. ITC analysis of ligand binding to preQ $_{1}$ riboswitches. Methods Enzymol 549, 435-50 (2014).

31. Coppins, R.L., Hall, K.B. \& Groisman, E.A. The intricate world of riboswitches. Current opinion in microbiology 10, 176-181 (2007).

32. Ray, S., Chauvier, A. \& Walter, N.G. Kinetics coming into focus: single-molecule microscopy of riboswitch dynamics. RNA Biology 16, 1077-1085 (2019).

33. Lund, P.E., Chatterjee, S., Daher, M. \& Walter, N.G. Protein unties the pseudoknot: S1mediated unfolding of RNA higher order structure. Nucleic Acids Res (2019).

34. Suddala, K.C. \& Walter, N.G. Riboswitch structure and dynamics by smFRET microscopy. Methods Enzymol 549, 343-73 (2014).

35. Rio, D.C. Filter-binding assay for analysis of RNA-protein interactions. Cold Spring Harb Protoc 2012, 1078-81 (2012).

36. Dorywalska, M. et al. Site-specific labeling of the ribosome for single-molecule spectroscopy. Nucleic Acids Res 33, 182-9 (2005).

37. Blanchard, S.C., Kim, H.D., Gonzalez, R.L., Jr., Puglisi, J.D. \& Chu, S. tRNA dynamics on the ribosome during translation. Proc Natl Acad Sci U S A 101, 12893-8 (2004).

38. Francis, K. \& Kohen, A. Standards for the reporting of kinetic isotope effects in enzymology. Perspectives in Science 1, 110-120 (2014).

39. Li, A., Ziehr, J.L. \& Johnson, K.A. A new general method for simultaneous fitting of temperature and concentration dependence of reaction rates yields kinetic and 
thermodynamic parameters for HIV reverse transcriptase specificity. J Biol Chem 292, 66956702 (2017).

40. Spitzer, J. \& Poolman, B. The Role of Biomacromolecular Crowding, lonic Strength, and Physicochemical Gradients in the Complexities of Life's Emergence. Microbiology and Molecular Biology Reviews 73, 371-388 (2009).

41. $\mathrm{Qu}, \mathrm{X}$. et al. Single-molecule nonequilibrium periodic $\mathrm{Mg}^{2+}$-concentration jump experiments reveal details of the early folding pathways of a large RNA. Proceedings of the National Academy of Sciences of the United States of America 105, 6602-6607 (2008).

42. Nakagawa, S., Niimura, Y., Miura, K.-i. \& Gojobori, T. Dynamic evolution of translation initiation mechanisms in prokaryotes. Proceedings of the National Academy of Sciences 107, 6382-6387 (2010).

43. Omotajo, D., Tate, T., Cho, H. \& Choudhary, M. Distribution and diversity of ribosome binding sites in prokaryotic genomes. BMC Genomics 16, 604 (2015).

44. Romero, D.A. et al. A comparison of key aspects of gene regulation in Streptomyces coelicolor and Escherichia coli using nucleotide-resolution transcription maps produced in parallel by global and differential RNA sequencing. Mol Microbiol (2014).

45. Beck, H.J. \& Moll, I. Leaderless mRNAs in the Spotlight: Ancient but Not Outdated! Microbiol Spectr 6(2018).

46. Duval, M. et al. Escherichia coli ribosomal protein S1 unfolds structured mRNAs onto the ribosome for active translation initiation. PLoS Biol 11, e1001731 (2013).

47. Studer, S.M. \& Joseph, S. Unfolding of mRNA secondary structure by the bacterial translation initiation complex. Mol Cell 22, 105-15 (2006).

48. Salah, P. et al. Probing the relationship between Gram-negative and Gram-positive S1 proteins by sequence analysis. Nucleic Acids Res 37, 5578-88 (2009).

49. Fei, J. et al. Chapter 12 - A Highly Purified, Fluorescently Labeled In Vitro Translation System for Single-Molecule Studies of Protein Synthesis. in Methods in Enzymology, Vol. 472 (ed. Walter, N.G.) 221-259 (Academic Press, 2010).

50. Tsai, A. et al. Heterogeneous pathways and timing of factor departure during translation initiation. Nature 487, 390-393 (2012).

51. MacDougall, D.D. \& Gonzalez, R.L., Jr. Translation initiation factor 3 regulates switching between different modes of ribosomal subunit joining. J Mol Biol 427, 1801-18 (2015).

52. Wang, J., Caban, K. \& Gonzalez, R.L., Jr. Ribosomal initiation complex-driven changes in the stability and dynamics of initiation factor 2 regulate the fidelity of translation initiation. Journal of molecular biology 427, 1819-1834 (2015).

53. Saito, K., Green, R. \& Buskirk, A.R. Translational initiation in E. coli occurs at the correct sites genome-wide in the absence of mRNA-rRNA base-pairing. Elife 9(2020).

54. Tollerson, R., 2nd \& Ibba, M. Translational regulation of environmental adaptation in bacteria. J Biol Chem 295, 10434-10445 (2020).

55. Suddala, K.C. et al. Local-to-global signal transduction at the core of a $\mathrm{Mn}(2+)$ sensing riboswitch. Nat Commun 10, 4304 (2019).

56. Kozak, M. Regulation of translation via mRNA structure in prokaryotes and eukaryotes. Gene 361, 13-37 (2005). 
57. Del Campo, C., Bartholomaus, A., Fedyunin, I. \& Ignatova, Z. Secondary Structure across the Bacterial Transcriptome Reveals Versatile Roles in mRNA Regulation and Function. PLoS Genet 11, e1005613 (2015).

58. Passalacqua, K.D. et al. Structure and complexity of a bacterial transcriptome. J Bacterio/ 191, 3203-11 (2009).

59. Chiaruttini, C. \& Guillier, M. On the role of mRNA secondary structure in bacterial translation. Wiley Interdiscip Rev RNA, e1579 (2019).

60. Bastet, L. et al. Translational control and Rho-dependent transcription termination are intimately linked in riboswitch regulation. Nucleic Acids Res 45, 7474-7486 (2017).

61. Azam, M.S. \& Vanderpool, C.K. Translational regulation by bacterial small RNAs via an unusual Hfq-dependent mechanism. Nucleic Acids Res 46, 2585-2599 (2018).

62. Caron, M.-P. et al. Dual-acting riboswitch control of translation initiation and mRNA decay. Proceedings of the National Academy of Sciences of the United States of America 109, E3444E3453 (2012).

63. Leontis, N.B. \& Westhof, E. Geometric nomenclature and classification of RNA base pairs. RNA 7, 499-512 (2001). 


\section{Ray, et al., Figure 1}

a

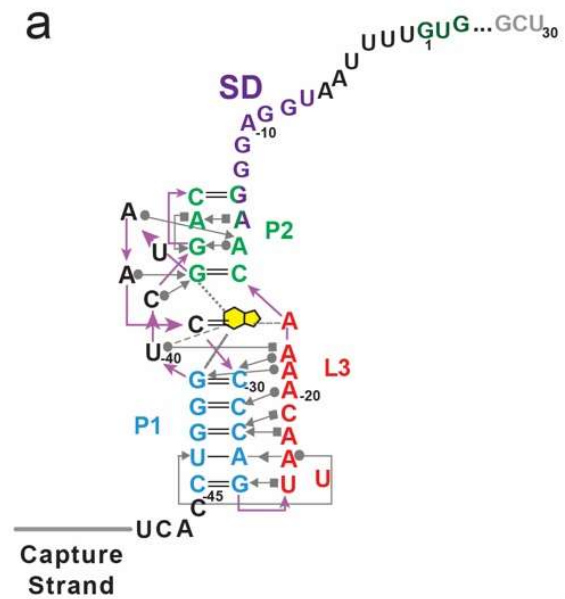

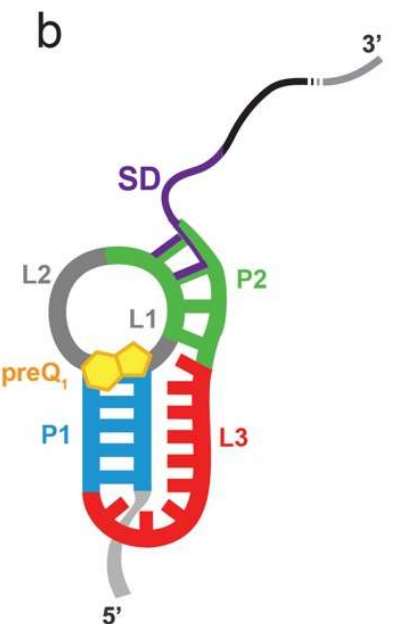

d

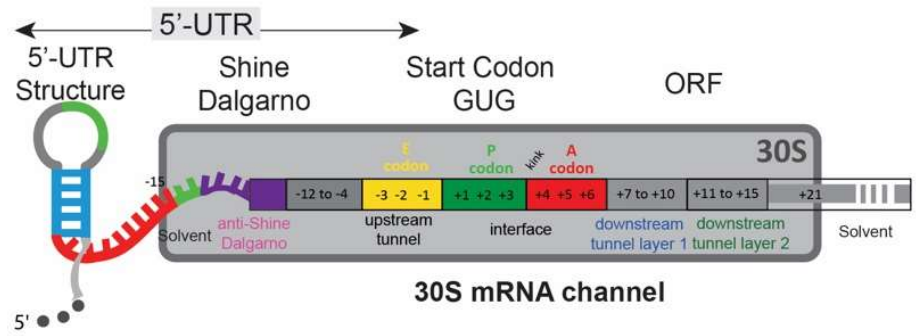

e
C

mRNA

H44 of 30 S

S1 protein

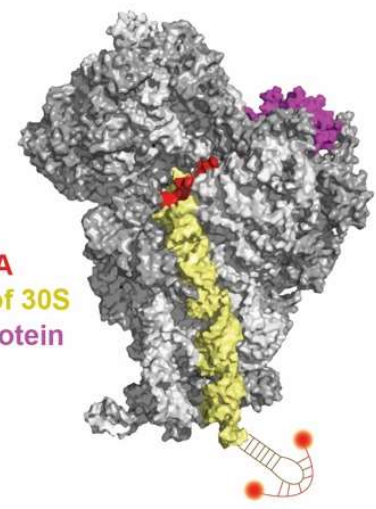

E. coli $30 \mathrm{~S}$ with $\mathrm{H} 44$ helix extension

\section{f}
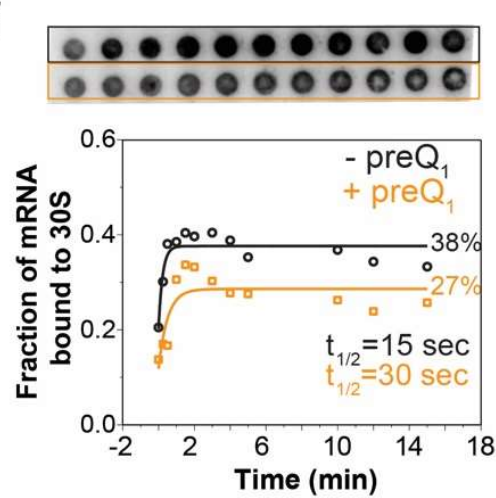

Figure 1: Schematic of a model mRNA with Tte-riboswitch at its 5'-UTR to study translation initiation. (a) Structural map of the preQ ${ }_{1}$ translational riboswitch from Tte displayed with Leontis-Westhof notations ${ }^{63}$. The SD sequence (purple) partially overlaps the P2 stem 
nucleotides (green). (b) Schematic diagram of the preQ 1 bound riboswitch containing mRNA (termed R-mRNA) from T. tengcongensis (Tte). (c) Schematic representation of the mutated ribosome used here, containing a hairpin extension at the helix-44. The extension is hybridized with a dual-labeled complementary DNA oligonucleotide (red). (d) Schematic diagram of Tte R-mRNA and its expected occupancy in the mRNA channel of the 30 s subunit during initiation, where +1 is the first nucleotide of the open reading frame. The mRNA channel representation is adapted from Ref. 12. (e) Base-pairing interactions between the Shine-Dalgarno sequence of our Tte mRNA transcript and the $16 \mathrm{~S}$ rRNA of E. coli or Tte. (f) Autoradiograph of a filter binding membrane measuring the efficiency of 30S-mRNA complex formation as a function of time at zero and $10 \mu \mathrm{M}$ preQ ${ }_{1}$ concentrations (top). The resulting fraction of bound mRNA is plotted as a function of time (bottom). 


\section{Ray, et al., Figure 2}
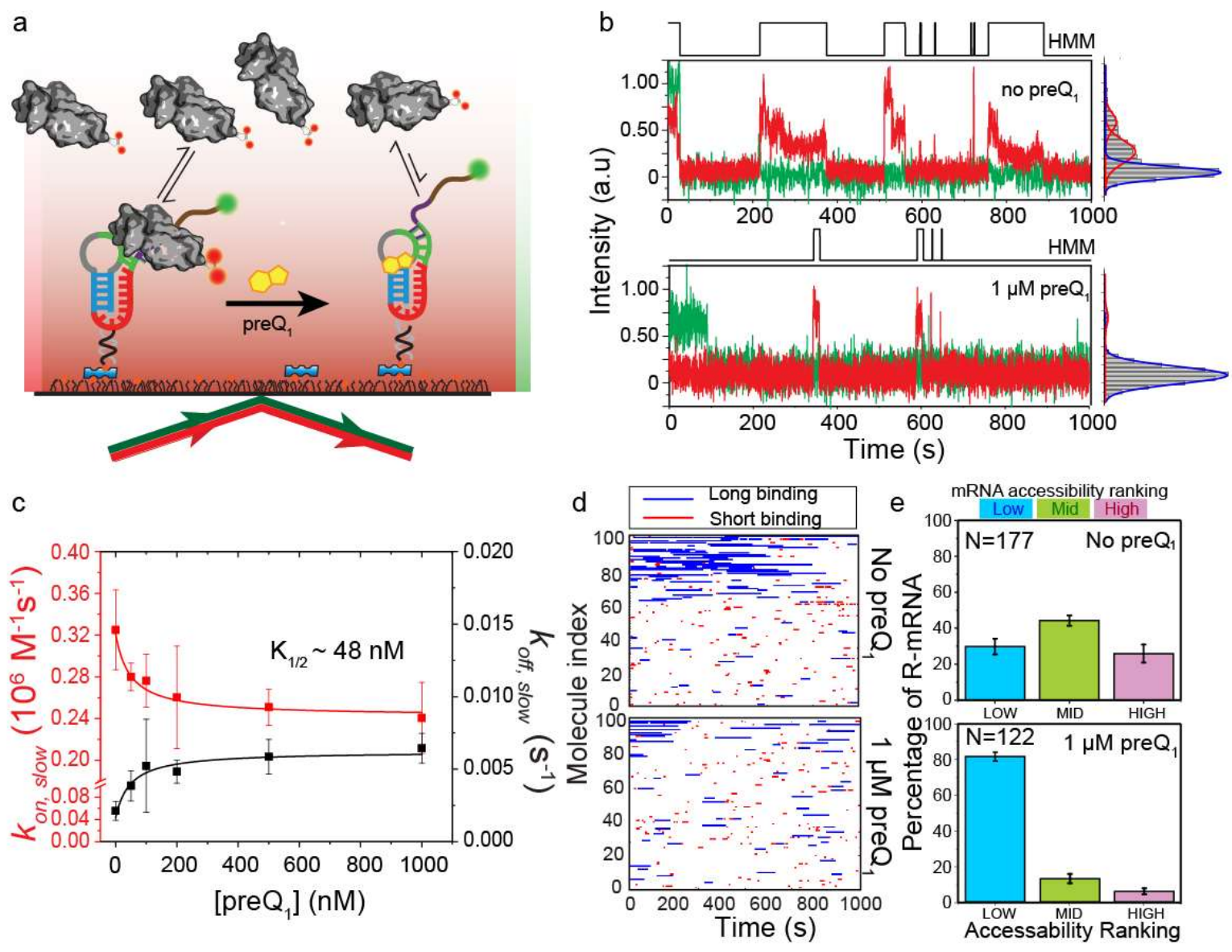

Figure 2: preQ ${ }_{1}$-dependent $30 S$ binding to R-mRNA at the single-molecule level. (a) Schematic representation of our SiM-KARB assay. 3'-Cy3 labeled R-mRNA molecules are immobilized on a slide surface. Repeated binding and dissociation of 30 S subunits (dual-labeled with Cy5) is monitored through the co-localization of Cy3 and Cy5 fluorescence. (b) Representative R-mRNA ${ }^{+30}$ (green) and 305 (red) fluorescence intensity versus time trajectories and corresponding intensity histograms for a single R-mRNA ${ }^{+30}$ molecule in the absence (top panel) or presence (bottom panel) of preQ ${ }_{1}$. $\mathrm{HMM}$ idealizations to a two-state model (black, top) are plotted as a function of time. (c) 30S-binding ( $k_{\text {on,slow, }}$ red) and dissociation ( $k_{\text {off,slow; }}$ black) rate constants were determined from exponential fits of 
dwell times in the unbound and bound states, respectively, as a function of $p \mathrm{Q}_{1}$ concentration. The corresponding half-saturation $\left(\mathrm{K}_{1 / 2}\right)$ values were determined by Hill equation fitting with Hill coefficients of -1 and +1 , respectively. (d) Rastergram of 100 randomly selected traces of 30 Sinding to individual $\mathrm{R}-\mathrm{mRNA}^{+30}$ molecules in the absence (top panel) and presence (bottom panel) of preQ ${ }_{1}$. $30 \mathrm{~S}$ binding events on $\mathrm{R}-\mathrm{mRNA}^{+30}$ are represented in either red (short or standby events) and blue (long or cleft-accommodated events). (e) Histograms of the fractional bound time for which individual R-mRNA ${ }^{+30}$ molecules are occupied by 30 S subunits in the absence (top) or presence (bottom) of preQ $_{1}$. All 30S-bound R-mRNA ${ }^{+30}$ molecules were empirically ranked as low $(<0.1$ fractional bound time, cyan), mid (0.1 to 0.2 fractional bound-time, green), or high (>0.2 fractional bound time, pink) in 30 S occupancy. $\mathrm{N}$ represents the total number of molecules included for each condition. 


\section{Ray, et al., Figure 3}
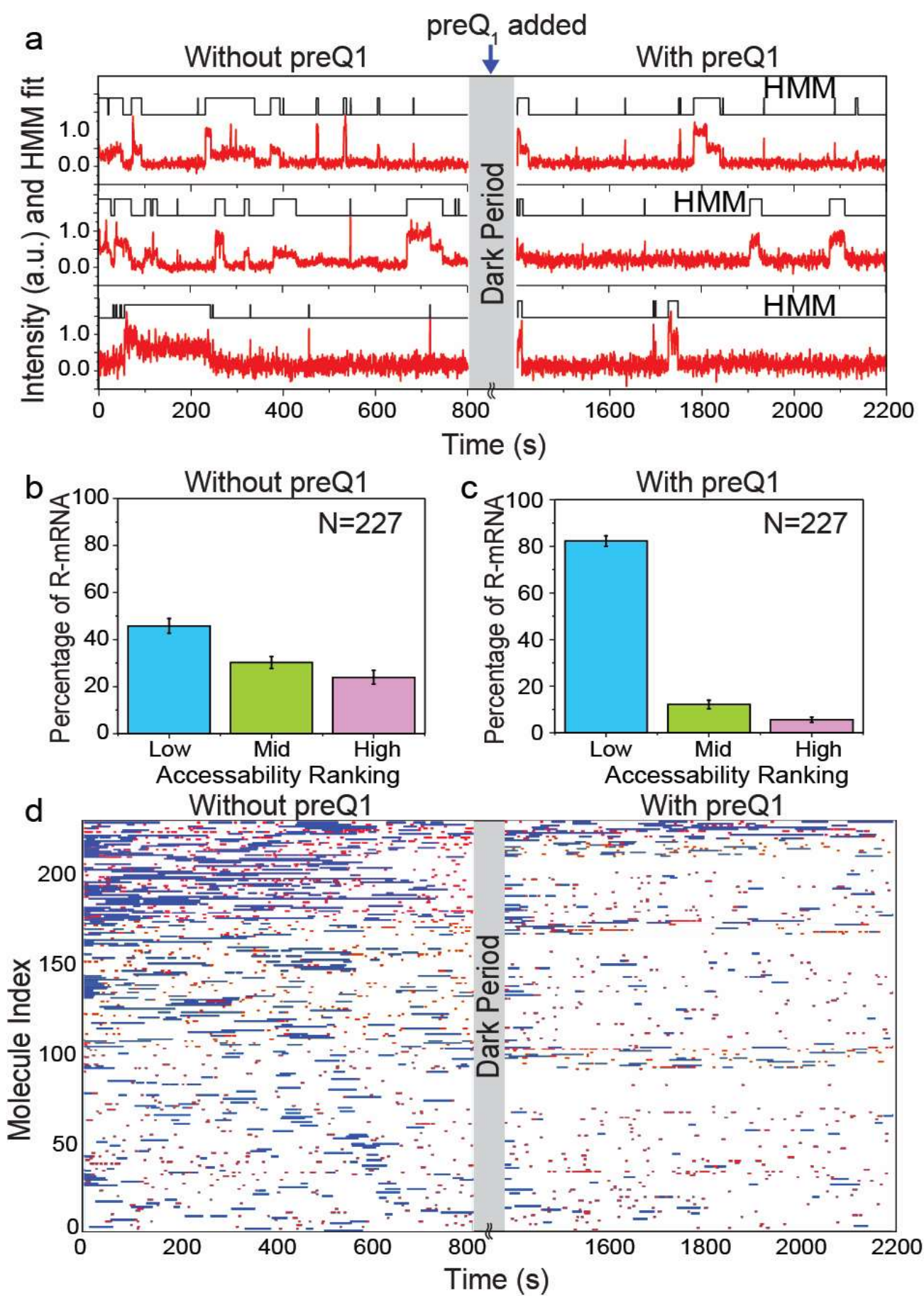

Figure 3: Single mRNA molecules undergo conformational switching upon introduction of preQ $_{1}$

ligand in situ. (a) Exemplary single-molecule trajectories from non-equilibrium ligand-jump 
experiments composed of segments before and after the jump. 30 S binding to the same set of individual $R-m R N A^{+30}$ was monitored first in the absence of preQ $_{1}$ (left, - preQ $_{1}$ ), then in the presence of $1 \mu \mathrm{M}$ preQ ${ }_{1}$ (right, $+\mathrm{preQ}_{1}$ ). The gray axis break represents a $600 \mathrm{sec}$ dark period between segments during which the buffer was exchanged. $(b, c)$ Histograms of the fraction of time individual R-mRNA ${ }^{+30}$ molecules are bound by $30 S$ before (b) and after (b) preQ $_{1}$ is introduced. Most molecules shift towards lower occupancy upon introduction of preQ ${ }_{1}$. (d) Rastergram displaying the 30 S binding behaviors of individual R-mRNA ${ }^{+30}$ molecules before and after introducing preQ ${ }_{1} .30 \mathrm{~S}$ binding events on each $\mathrm{R}-\mathrm{mRNA}^{+30}$ are represented in red (short or standby events) or blue bar (long or cleftaccommodated events). 


\section{Ray, et al., Figure 4}

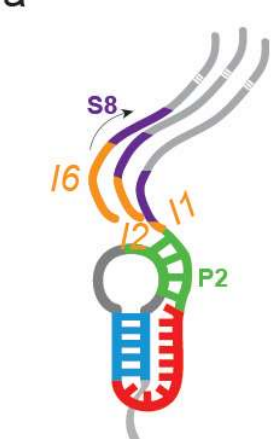

SD-Aptamer distance varied

e

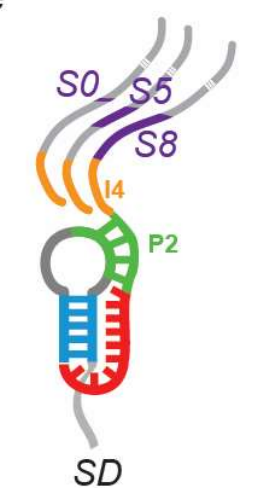

length varied b

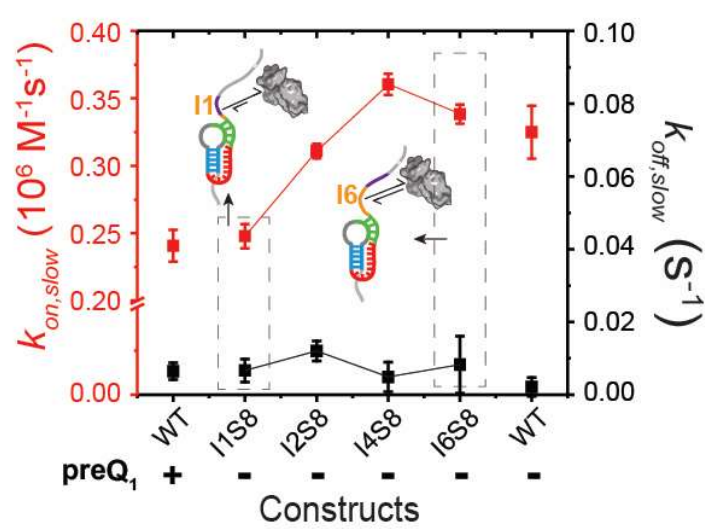

f

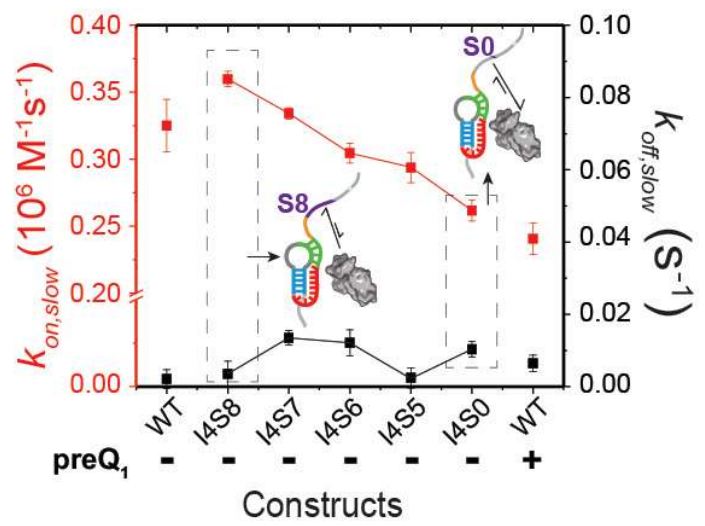

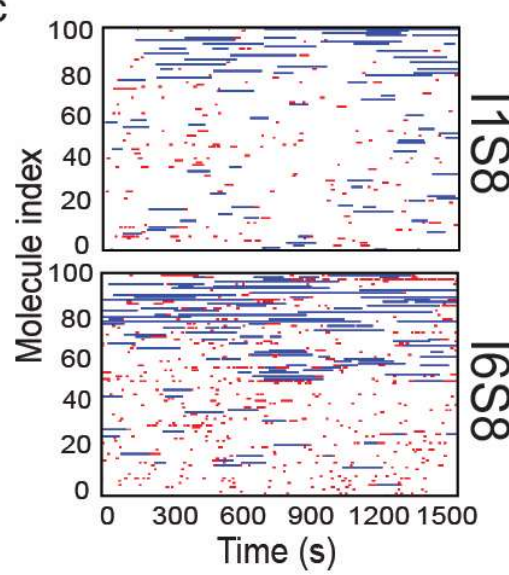

g

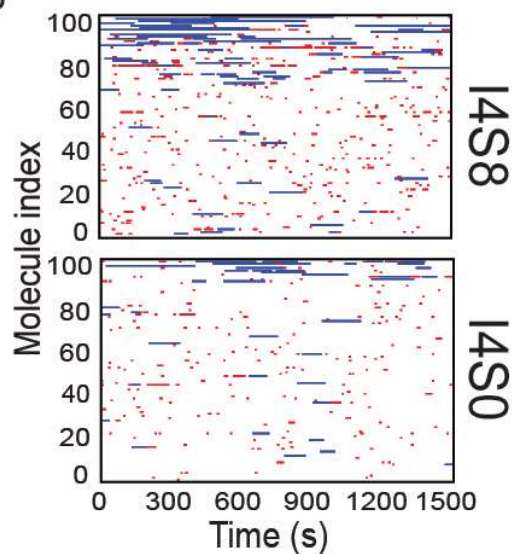

d

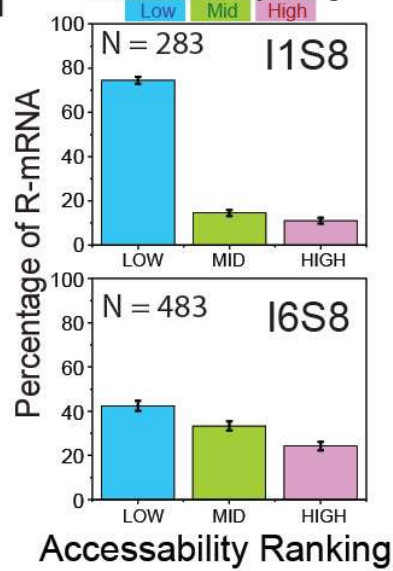

Accessability Ranking

h

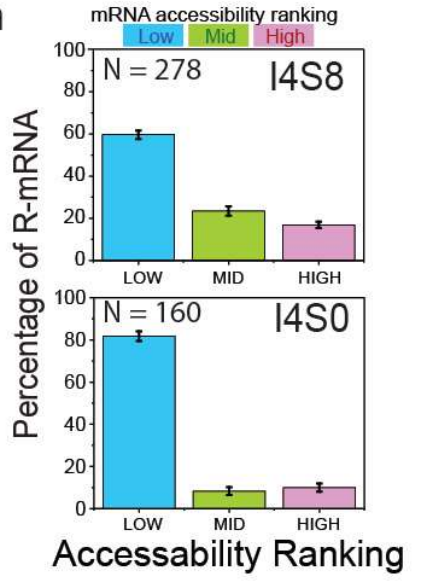

Figure 4: Mutation of the 5'-UTR structure by varying SD-aptamer distance (I) and SD length (S) affects 30S binding. (a) Schematic

representation for varying the SD-aptamer distance from insert length of one base (I1) to six bases (I6). (b) 30S-binding ( $k_{\text {on,slow, }}$ red) and 


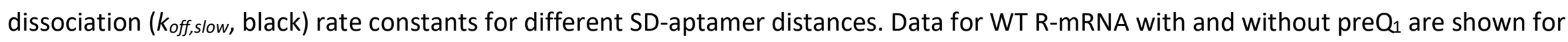
reference. (c) Rastergram of 100 randomly selected traces of individual mutant R-mRNA ${ }^{+30}$ molecules with a 1-nt (I1S8, top panel) or 6base insert (I6S8, bottom panel). 30S binding is represented in red (short binding events or standby events) or blue (long binding events or cleft-accommodated events). (d) Histogram of the fractional bound time for individual I1S8 (top) and I6S8 (bottom) mutant R-mRNA molecules. (e) Schematic representation for varying the SD:anti-SD interaction length from full complementarity (S8) to no complementarity (SO) with a fixed number of $4 \mathrm{nt}(\mathrm{I})$ between the aptamer and SD sequence. (f) $30 \mathrm{~S}$ binding ( $k_{\text {on, slow, }}$ red) and dissociation ( $k_{\text {off,slow, }}$ black) rate constants for different SD:anti-SD complementarities. (g) Rastergram of 100 randomly selected traces of individual mutant R-mRNA ${ }^{+30}$ molecules with fully available SD (14S8, top panel) or no SD (I4S0, bottom panel). (h) Histogram of the fractional bound time for individual 14 S8 (top) and I4SO (bottom) mutant R-mRNA molecules. 


\section{Ray, et al., Figure 5}
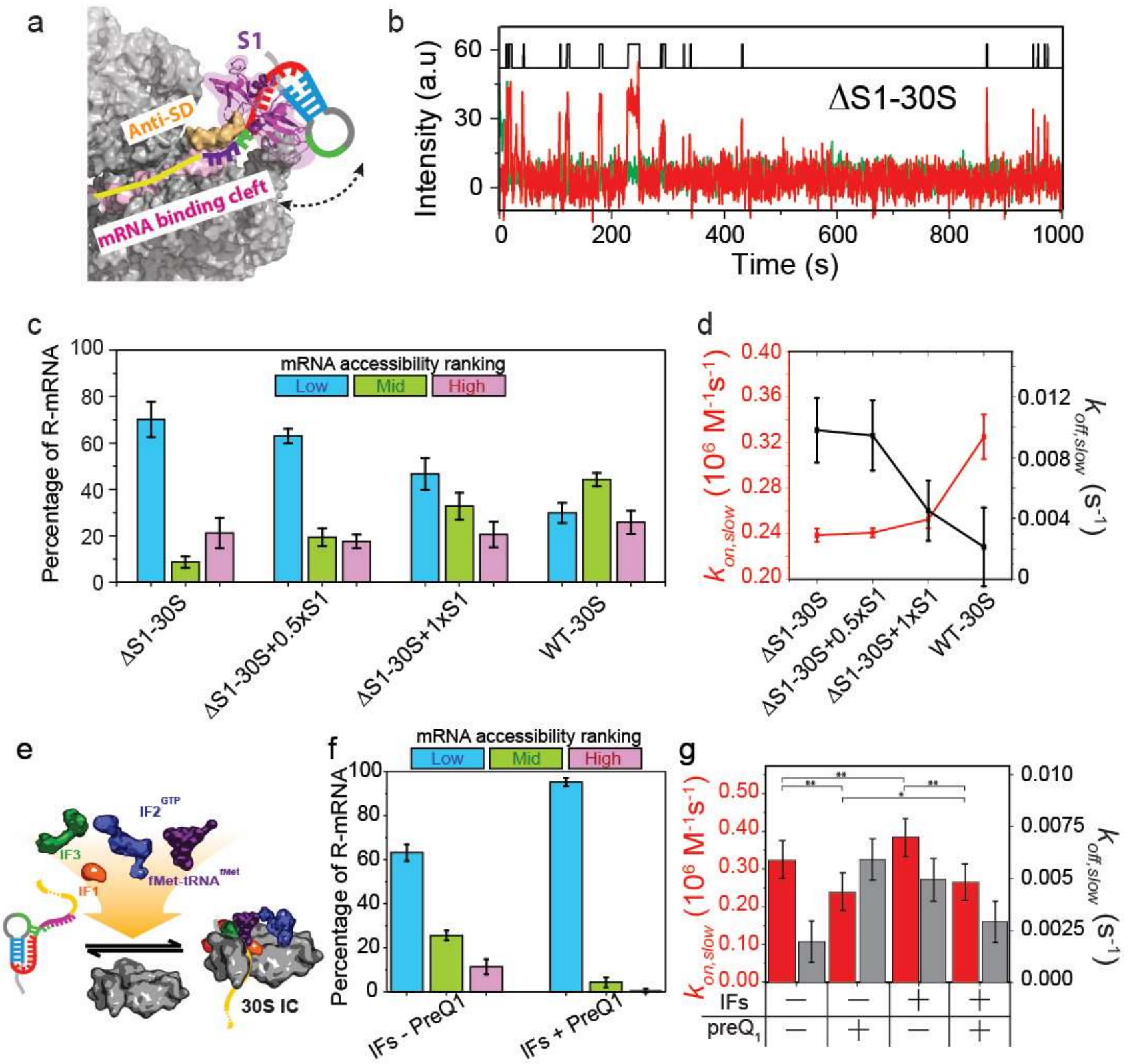

Figure 5: S1 mediates unfolding of the 5'-UTR to enhance and stabilize 30 S binding. (a) Schematic of

S1 mediated unfolding of the $5^{\prime}$-UTR of R-mRNA ${ }^{+30}$. (b) Representative R-mRNA ${ }^{+30}$ (green) and associated $\triangle \mathrm{S} 1-30 \mathrm{~S}$ (red) binding time trajectory. An HMM idealization to a two-state model (black, top) is plotted as a function of time. (c) Histogram of the fractional bound time for individual R-mRNA 
molecules occupied by for depleted $\Delta \mathrm{S} 1-30 \mathrm{~S}, \Delta \mathrm{S} 1-30 \mathrm{~S}$ with $50 \%$ stoichiometric purified S1 $(\Delta \mathrm{S} 1-$ $30 \mathrm{~S}+0.5 \times \mathrm{S} 1), \Delta \mathrm{S} 1-30 \mathrm{~S}$ with fully stoichiometric purified S1 $(\Delta \mathrm{S} 1-30 \mathrm{~S}+1 \times \mathrm{S} 1)$, and WT-30S. (d) Corresponding binding $\left(k_{o n, s l o w}\right.$, red) and dissociation $\left(k_{\text {off,slow }}\right.$, black) rate constants. Data for WT RmRNA without preQ $_{1}$ are shown for comparison. (e) Schematic of pre-initiation complex formation in the presence of initiation factors and initiator tRNA. (f) Histogram of the fractional bound-time for individual R-mRNA ${ }^{+30}$ in the presence of all initiation factors and fMet-tRNA ${ }^{f M e t}$, in the absence (top) and presence (bottom) of preQ $_{1}$. $(\mathrm{g})$ Comparison of binding $\left(k_{\text {on, slow }}\right)$ and dissociation $\left(k_{\text {off,slow }}\right)$ rate constants in the presence or absence of initiation factors fMet-tRNA ${ }^{\text {fmet }}$ and preQ ${ }_{1}$, as indicated. Statistical significance was determined by student t-test as $* p<10^{-2} ; * * p<10^{-4}$. 


\section{Ray, et al., Figure 6}

a

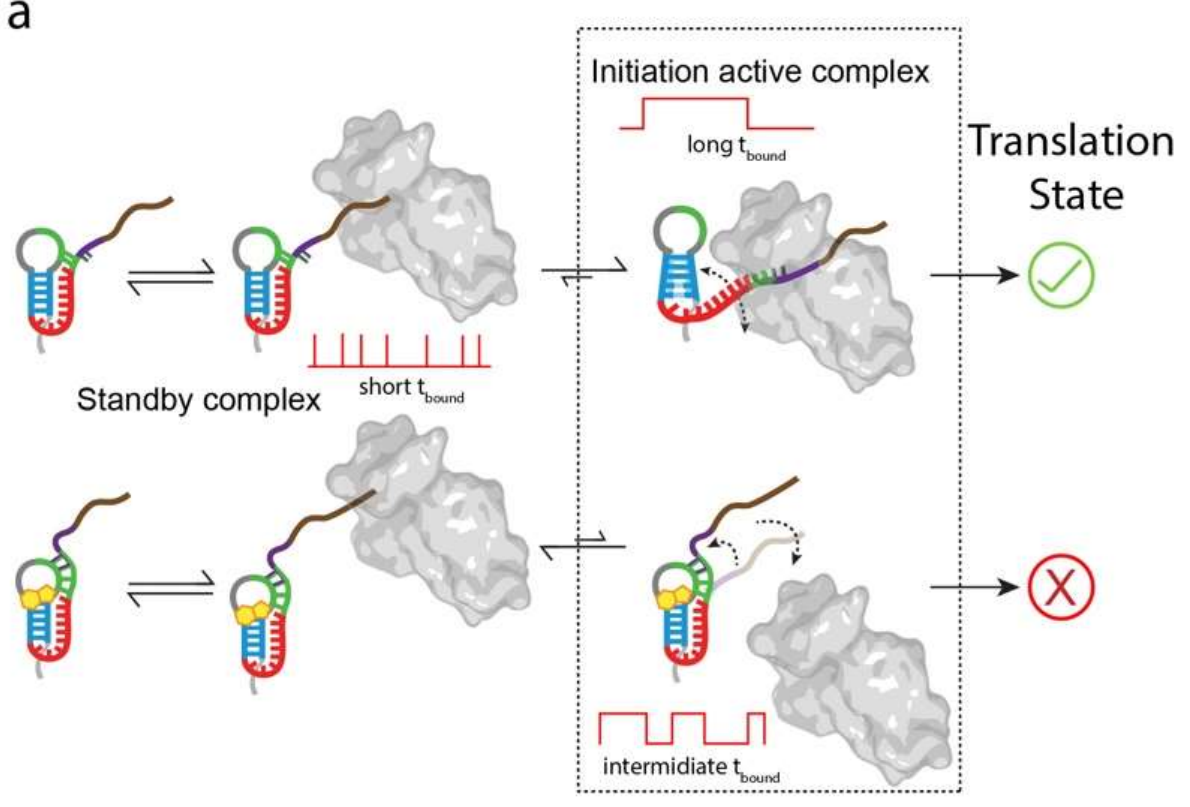

b

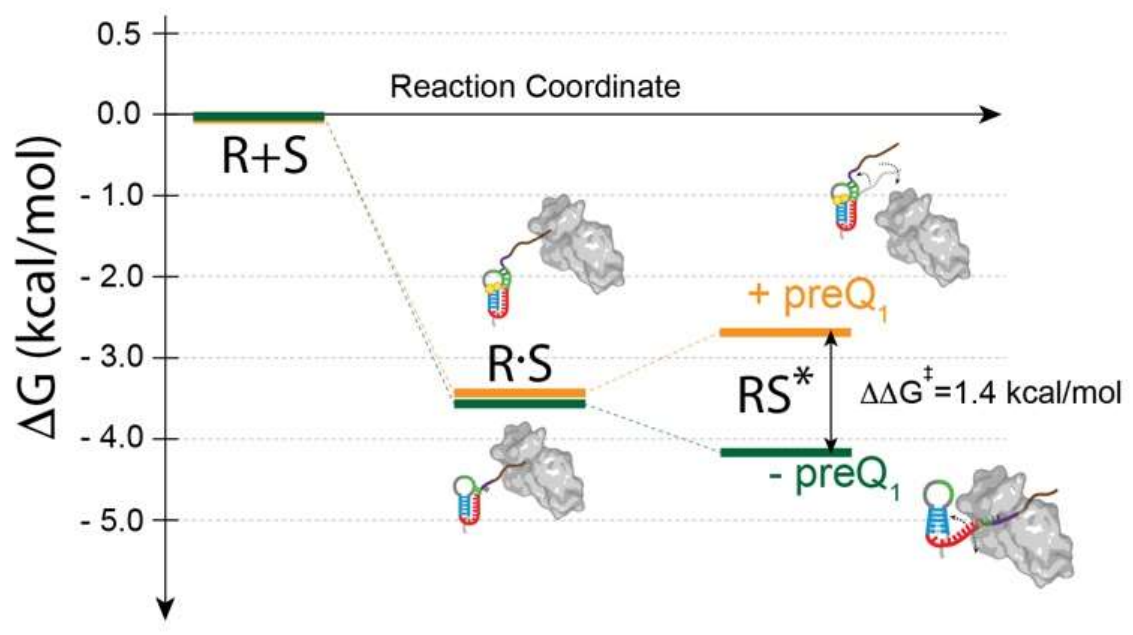

Figure 6: Model for the earliest stage of translation initiation in dependence of an embedded riboswitch structure. (a) Independent of

the preQ $_{1}$, the $30 S$ dynamically interacts with the R-mRNA scanning for the RBS (and SD sequence) to facilitate translation initiation on the nascent mRNA. Short, transient 30S binding is categorized as standby binding, whereas long binding events represent mRNA accommodation with established SD:anti-SD interactions, required to initiate translation. In the absence of preQ ${ }_{1}$, the SD region is more accessible, favoring complete accommodation of the mRNA through correct SD:anti-SD interactions and more stable 30S binding. By 
contrast, in the presence of preQ $_{1}$ the SD sequence is partially sequestered, leading to more standby site binding of the mRNA on the $30 \mathrm{~S}$ subunit and to more frequently disrupted mRNA accommodation, both resulting in unsuccessful translation initiation. (b) Free energy diagram calculated from the $30 \mathrm{~S}$ binding and dissociation rate constants to $\mathrm{R}-\mathrm{mRNA}^{+30}$ in the absence or presence of preQ ${ }_{1}$. An energetic penalty of $\sim 1.4 \mathrm{kcal} / \mathrm{mol}$ for formation of the mRNA cleft accommodated complex derives from the dynamic unfolding of the $5^{\prime}$-UTR, necessary for a complete accommodation of the mRNA into the 305 subunit. 


\section{Supplementary Files}

This is a list of supplementary files associated with this preprint. Click to download.

- RayetalSupportinglnfo.pdf 